\title{
A Generalized Ratio-cum-Product Estimator of Finite Population Mean in Stratified Random Sampling
}

\author{
Rajesh Tailor ${ }^{a}$, Balkishan Sharma ${ }^{b}$, Jong-Min Kim $^{1, c}$ \\ ${ }^{a}$ School of Studies in Statistics, Vikram University; ${ }^{b}$ Aurobindo Institute of Medical Science \\ ${ }^{c}$ Statistics, Division of Science and Mathematics, University of Minnesota- Morris
}

\begin{abstract}
This paper suggests a ratio-cum product estimator of a finite population mean using information on the coefficient of variation and the fcoefficient of kurtosis of auxiliary variate in stratified random sampling. Bias and MSE expressions of the suggested estimator are derived up to the first degree of approximation. The suggested estimator has been compared with the combined ratio estimator and several other estimators considered by Kadilar and Cingi (2003). In addition, an empirical study is also provided in support of theoretical findings.

Keywords: Finite population mean, coefficient of variation, correlation coefficient, stratified random sampling, bias, mean squared error.
\end{abstract}

\section{Introduction}

The problem of the estimation of population parameters like mean, variance, and ratio of two population means are common in agriculture, economics, medicine, and population studies. The use of auxiliary information has been applied for improving the efficiencies of the estimators of population parameter $(s)$ irrespective of sampling design. Ratio, product and regression methods of estimation are good examples in this context. Cochran (1940) used auxiliary information at the estimation stage and proposed a ratio estimator for the population mean. A ratio estimator is preferred when the correlation coefficient between the study variate and the auxiliary variate is positive. Robson (1957) defined a product estimator that was revisited by Murthy (1964). The product estimator is used when the correlation between the study variate and the auxiliary is negative.

Sisodia and Dwivedi (1981), Pandey and Dubey (1988), Upadhyaya and Singh (1999), Singh et al. (2004) and Singh and Tailor (2005) later used known values of various parameters of an auxiliary variate in simple random sampling.

A combined ratio and product are some basic estimators of the population mean that uses information on the auxiliary variate in stratified random sampling. Kadilar and Cingi (2003) defined, Sisodia and Dwivedi (1981), Upadhyaya and Singh (1999) and Singh et al. (2004) estimators in stratified random sampling.

Singh and Espejo (2003) suggested a ratio-cum-product estimator using scalar $\alpha$. This estimator posses a nice property that for $\alpha=1$, it becomes useful for a positive correlation while for $\alpha=0$, it is quite effective in a negative correlation.

Singh and Tailor (2005) and Tailor and Sharma (2009) proposed ratio-cum-product estimator of a finite population mean in simple random sampling. Tailor (2009) defined a ratio-cum product estimator in stratified random sampling.

\footnotetext{
${ }^{1}$ Corresponding author: Statistics, Division of Science and Mathematics, University of Minnesota- Morris, MN 5627, USA. E-mail: jongmink@morris.umn.edu
} 
Researchers are motivated to work in this direction and we propose a ratio-cum-product estimator using information on the coefficient of variation and the coefficient of the kurtosis of auxiliary variate in stratified random sampling.

Let $U=\left(U_{1}, U_{2}, \ldots, U_{N}\right)$ be a finite population of size $N$ and it is divided into $k$ homogeneous strata of size $N_{h}(h=1,2, \ldots, k)$. A sample of size $n_{h}$ is drawn from each stratum using the simple random sampling without replacement.

Let $y$ be the study variate taking values $y_{h i}\left(i^{\text {th }}\right.$ observation from $h^{\text {th }}$ stratum) and $x_{h i}$ be the auxiliary variate taking values $x_{h i}$.

Let $\bar{y}_{s t}=\sum_{h=1}^{k} W_{h} \bar{y}_{h}$ and $\bar{x}_{s t}=\sum_{h=1}^{k} W_{h} \bar{x}_{h}$ be the unbiased estimators of the population mean $\bar{Y}$ and $\bar{X}$ of the study variate and auxiliary variate respectively, where

$$
\begin{aligned}
W_{h} & =\frac{N_{h}}{N} \quad: \text { is the weight of } h^{\text {th }} \text { stratum, } \\
\bar{y}_{h} & =\frac{1}{n_{h}} \sum_{j=1}^{n_{h}} \bar{y}_{h j} \quad: \text { sample mean of the study variate } y \text { for } h^{\text {th }} \text { stratum, } \\
\bar{x}_{h} & =\frac{1}{n_{h}} \sum_{j=1}^{n_{h}} \bar{x}_{h j} \quad: \text { sample mean of the auxiliary variate } x \text { for } h^{\text {th }} \text { stratum. }
\end{aligned}
$$

The usual combined ratio and product estimators of population mean $\bar{Y}$ respectively are

$$
\begin{aligned}
& \hat{\bar{Y}}_{R S T}=\bar{y}_{s t}\left(\frac{\bar{X}}{\bar{x}_{s t}}\right), \\
& \hat{\bar{Y}}_{P S T}=\bar{y}_{s t}\left(\frac{\bar{x}_{s t}}{\bar{X}}\right) .
\end{aligned}
$$

The mean squared error(MSE) expressions of the combined ratio and product estimators up to the first degree of approximation are

$$
\begin{aligned}
& \operatorname{MSE}\left(\hat{\bar{Y}}_{R S T}\right)=\sum_{h=1}^{k} W_{h}^{2} \gamma_{h}\left(S_{y h}^{2}+R^{2} S_{x h}^{2}-2 R S_{y x h}\right), \\
& \operatorname{MSE}\left(\hat{\bar{Y}}_{P S T}\right)=\sum_{h=1}^{k} W_{h}^{2} \gamma_{h}\left(S_{y h}^{2}+R^{2} S_{x h}^{2}+2 R S_{y x h}\right),
\end{aligned}
$$

where

$$
\begin{aligned}
& R=\frac{\bar{Y}}{\bar{X}}, \quad \gamma_{h}=\left(\frac{N_{h}-n_{h}}{N_{h} n_{h}}\right), \quad S_{y h}^{2}=\frac{1}{N_{h}-1} \sum_{j=1}^{N_{h}}\left(y_{h j}-\bar{Y}_{h}\right)^{2}, \quad S_{x h}^{2}=\frac{1}{N_{h}-1} \sum_{j=1}^{N_{h}}\left(x_{h j}-\bar{X}_{h}\right)^{2} \\
& \text { and } \quad S_{y x h}=\frac{1}{N_{h}-1} \sum_{j=1}^{N_{h}}\left(y_{h j}-\bar{Y}_{h}\right)\left(x_{h j}-\bar{X}_{h}\right) .
\end{aligned}
$$

Sisodia and Dwivedi (1981) suggested a ratio estimator of population mean $\bar{Y}$ using the coefficient of variation of auxiliary variate $\left(C_{x}\right)$ as

$$
\hat{\bar{Y}}_{S D}=\bar{y}\left[\frac{\bar{X}+C_{x}}{\bar{x}+C_{x}}\right]
$$


Singh et al. (2004) proposed another ratio estimator for $\bar{Y}$, using the coefficient of kurtosis $\left(\beta_{2}(x)\right)$ of the auxiliary variate $x$ as

$$
\hat{\bar{Y}}_{S E}=\bar{y}\left[\frac{\bar{X}+\beta_{2}(x)}{\bar{x}+\beta_{2}(x)}\right],
$$

Upadhyaya and Singh (1999) used information on the coefficient of variation $\left(C_{x}\right)$ and the coefficient of the kurtosis $\left(\beta_{2}(x)\right)$ of the auxiliary variate $x$ and suggested two estimators for $\bar{Y}$

$$
\hat{\bar{Y}}_{U S 1}=\bar{y}\left[\frac{\bar{X} \beta_{2}(x)+C_{x}}{\bar{x} \beta_{2}(x)+C_{x}}\right]
$$

and

$$
\hat{\bar{Y}}_{U S 2}=\bar{y}\left[\frac{\bar{x} C_{x}+\beta_{2}(x)}{\bar{X} C_{x}+\beta_{2}(x)}\right],
$$

Kadilar and Cingi (2003) defined $\hat{\bar{Y}}_{S D}, \hat{\bar{Y}}_{S E}, \hat{\bar{Y}}_{U S 1}$ and $\hat{\bar{Y}}_{U S 2}$ in stratified random sampling respectively as

$$
\begin{gathered}
\hat{\bar{Y}}_{S D}^{S T}=\bar{y}_{s t}\left[\frac{W_{h}\left(\bar{X}_{h}+C_{x h}\right)}{\sum_{h=1}^{k} W_{h}\left(\bar{x}_{h}+C_{x h}\right)}\right], \\
\hat{\bar{Y}}_{S E}^{S T}=\bar{y}_{s t}\left[\frac{\sum_{h=1}^{k} W_{h}\left(\bar{X}_{h}+\beta_{2 h}(x)\right)}{\sum_{h=1}^{k} W_{h}\left(\bar{x}_{h}+\beta_{2 h}(x)\right)}\right], \\
\hat{\bar{Y}}_{U S 1}^{S T}=\bar{y}_{s t}\left[\frac{\sum_{h=1}^{k} W_{h}\left(\bar{X}_{h} \beta_{2 h}(x)+C_{x h}\right)}{\sum_{h=1}^{k} W_{h}\left(\bar{x}_{h} \beta_{2 h}(x)+C_{x h}\right)}\right], \\
\hat{\bar{Y}}_{U S 2}^{S T}=\bar{y}_{s t}\left[\frac{\sum_{h=1}^{k} W_{h}\left(\bar{X}_{h} C_{x h}+\beta_{2 h}(x)\right)}{\sum_{h=1}^{k} W_{h}\left(\bar{x}_{h} C_{x h}+\beta_{2 h}(x)\right)}\right] .
\end{gathered}
$$

To the first degree of approximation mean squared errors of $\hat{\bar{Y}}_{S D}^{S T}, \hat{\bar{Y}}_{S E}^{S T}, \hat{\bar{Y}}_{U S 1}^{S T}$ and $\hat{\bar{Y}}_{U S 2}^{S T}$ respectively are

$$
\begin{aligned}
\operatorname{MSE}\left(\hat{\bar{Y}}_{S D}^{S T}\right) & =\sum_{h=1}^{k} W_{h}^{2} \gamma_{h}\left(S_{y h}^{2}+R_{S D}^{2} S_{x h}^{2}-2 R_{S D} S_{y x h}\right), \\
\operatorname{MSE}\left(\hat{\bar{Y}}_{S E}^{S T}\right) & =\sum_{h=1}^{k} W_{h}^{2} \gamma_{h}\left(S_{y h}^{2}+R_{S K}^{2} S_{x h}^{2}-2 R_{S K} S_{y x h}\right), \\
\operatorname{MSE}\left(\hat{\bar{Y}}_{U S 1}^{S T}\right) & =\sum_{h=1}^{k} W_{h}^{2} \gamma_{h}\left(S_{y h}^{2}+R_{U S 1}^{2} \beta_{2 h}^{2}(x) S_{x h}^{2}-2 R_{U S 1} \beta_{2 h}(x) S_{y x h}\right), \\
\operatorname{MSE}\left(\hat{\bar{Y}}_{U S 2}^{S T}\right) & =\sum_{h=1}^{k} W_{h}^{2} \gamma_{h}\left(S_{y h}^{2}+R_{U S 2}^{2} C_{x h}^{2} S_{x h}^{2}-2 R_{U S 2} C_{x h} S_{y x h}\right),
\end{aligned}
$$


where

$$
\begin{aligned}
R_{S D}=\bar{Y} / \sum_{h=1}^{k} W_{h}\left(\bar{X}_{h}+C_{x h}\right), & R_{S E}=\bar{Y} / \sum_{h=1}^{k} W_{h}\left(\bar{X}_{h}+\beta_{2 h}(x)\right), \\
R_{U S 1}=\bar{Y} / \sum_{h=1}^{k} W_{h}\left(\bar{X}_{h} \beta_{2 h}(x)+C_{x h}\right), & R_{U S 2}=\bar{Y} / \sum_{h=1}^{k} W_{h}\left(\bar{X}_{h} C_{x h}+\beta_{2 h}(x)\right) .
\end{aligned}
$$

\section{Suggested Ratio Estimator}

Assuming that the population coefficient of the variation and the coefficient of kurtosis are known for all the stratum, the suggested ratio-cum product estimator is

$$
\hat{\bar{Y}}_{b k}=\bar{y}_{s t}\left[\alpha\left\{\frac{\sum_{h=1}^{k} W_{h}\left(\bar{X}_{h} C_{x h}+\beta_{2 h}(x)\right)}{\sum_{h=1}^{k} W_{h}\left(\bar{x}_{h} C_{x h}+\beta_{2 h}(x)\right)}\right\}+(1-\alpha)\left\{\frac{\sum_{h=1}^{k} W_{h}\left(\bar{x}_{h} C_{x h}+\beta_{2 h}(x)\right)}{\sum_{h=1}^{k} W_{h}\left(\bar{X}_{h} C_{x h}+\beta_{2 h}(x)\right)}\right\}\right] .
$$

To obtain the bias and mean squared error of $\hat{\bar{Y}}_{b k}$, let $\bar{y}_{h}=\bar{Y}\left(1+e_{0 h}\right)$ and $\bar{x}_{h}=\bar{X}_{h}\left(1+e_{1 h}\right)$ such that

$$
E\left(e_{0 h}\right)=E\left(e_{1 h}\right)=0, \quad E\left(e_{0 h}^{2}\right)=\frac{1}{\bar{Y}_{h}^{2}} \gamma_{h} S_{y h}^{2}, \quad E\left(e_{1 h}^{2}\right)=\frac{1}{\bar{X}_{h}^{2}} \gamma_{h} S_{x h}^{2} \quad \text { and } \quad E\left(e_{0} e_{1}\right)=\frac{1}{\bar{Y}_{h} \bar{X}_{h}} \gamma_{h} S_{y x h}
$$

where $\gamma_{h}=\left(1 / n_{h}-1 / N_{h}\right)$.

Expressing (2.1) in terms of $e_{0 h}$ and $e_{1 h}$, we get

$$
\hat{\bar{Y}}_{b k}=\bar{Y}\left(1+e_{0}\right)\left\{\alpha\left(1+e_{1}\right)^{-1}+(1-\alpha)\left(1+e_{1}\right)\right\},
$$

where $e_{0}=1 / \bar{Y} \sum_{h=1}^{k} W_{h} \bar{Y}_{h} e_{0 h}$ and $e_{1}=\sum_{h=1}^{k} W_{h} a_{h} e_{1 h}$ here $a_{h}=\left(\bar{X}_{h} C_{x h}\right) /\left(\bar{X}_{U S 2}\right)$.

We now assume that $\left|e_{1}\right|<1$ so that we may expand $\left(1+e_{1}\right)^{-1}$ as a series in powers of $e_{1}$. To the first degree of approximation, the bias and mean squared error of the proposed estimator $\hat{\bar{Y}}_{b k}$ are

$$
\begin{aligned}
\operatorname{Bias}\left(\hat{\bar{Y}}_{b k}\right) & =\frac{1}{\bar{X}_{U S 2}} \sum_{h=1}^{k} W_{h}^{2} \gamma_{h} C_{x h}\left[\alpha R_{U S 2} C_{x h} S_{x h}^{2}+(1-2 \alpha) S_{y x h}\right], \\
\operatorname{MSE}\left(\hat{\bar{Y}}_{b k}\right) & =\sum_{h=1}^{k} W_{h}^{2} \gamma_{h}\left[S_{y h}^{2}+2 R_{U S 2}(1-2 \alpha) C_{x h} S_{y x h}+R_{U S 2}^{2}(1-2 \alpha)^{2} C_{x h}^{2} S_{x y}^{2}\right] .
\end{aligned}
$$

Mean squared error of $\hat{\bar{Y}}_{b k}$ is minimized for $\alpha=1 / 2\left[1+\left\{\sum_{h=1}^{k} W_{h}^{2} \gamma_{h} C_{x h} S_{y x h}\right\} /\left\{R_{U S 1} \sum_{h=1}^{k} W_{h}^{2} \gamma_{h} C_{x h}^{2}\right.\right.$ $\left.\left.S_{x h}^{2}\right\}\right]$.

By the substitution of $\alpha$ in (2.1) we get the asymptotically optimum estimator (AOE) for $\bar{Y}$ as

$$
\begin{aligned}
\hat{\bar{Y}}_{b k}^{(o p t)}= & \bar{y}_{s t} \\
& {\left[\frac{1}{2}\left(1+\frac{\sum_{h=1}^{k} W_{h}^{2} \gamma_{h} C_{x h} S_{y x h}}{R_{U S 2} \sum_{h=1}^{k} W_{h}^{2} \gamma_{h} C_{x h}^{2} S_{x h}^{2}}\right)\left(\frac{\sum_{h=1}^{k} W_{h}\left(\bar{X}_{h} C_{x h}+\beta_{2 h}(x)\right)}{\sum_{h=1}^{k} W_{h}\left(\bar{x}_{h} C_{x h}+\beta_{2 h}(x)\right)}\right)\right.} \\
& \left.\left\{1-\frac{1}{2}\left(1+\frac{\sum_{h=1}^{k} W_{h}^{2} \gamma_{h} C_{x h} S_{y x h}}{R_{U S 2} \sum_{h=1}^{k} W_{h}^{2} \gamma_{h} C_{x h}^{2} S_{x h}^{2}}\right)\right\}\left(\frac{\sum_{h=1}^{k} W_{h}\left(\bar{x}_{h} C_{x h}+\beta_{2 h}(x)\right)}{\sum_{h=1}^{k} W_{h}\left(\bar{X}_{h} C_{x h}+\beta_{2 h}(x)\right)}\right)\right] .
\end{aligned}
$$


Substituting the value of $\alpha$ in (2.4), minimum mean squared error of $\hat{\bar{Y}}_{b k}$ is

$$
\operatorname{MSE}\left(\hat{\bar{Y}}_{b k}^{o p t}\right)=\sum_{h=1}^{k} W_{h}^{2} \gamma_{h} S_{y h}^{2}-\frac{\left(\sum_{h=1}^{k} W_{h}^{2} \gamma_{h} C_{x h} S_{y x h}\right)^{2}}{\sum_{h=1}^{k} W_{h}^{2} \gamma_{h} C_{x h}^{2} S_{x h}^{2}}
$$

\section{Efficiency Comparisons}

The variance of the usual unbiased estimator of the mean $\bar{y}_{s t}$ in stratified random sampling is

$$
V\left(\bar{y}_{s t}\right)=\sum_{h=1}^{k} W_{h}^{2} \gamma_{h} S_{y h}^{2} .
$$

From (1.3), (1.13), (1.14), (1.15), (1.16), (2.4) and (3.1)

(i) $\operatorname{MSE}\left(\hat{\bar{Y}}_{b k}\right)<V\left(\bar{y}_{s t}\right)$ if

$$
\left.\begin{array}{ll}
\text { either } & \frac{1}{2}<\alpha<\left(\frac{1}{2}+\frac{B_{1}}{A_{1} R_{U S 2}}\right) \\
\text { or } & \left(\frac{1}{2}+\frac{B_{1}}{A_{1} R_{U S 2}}\right)<\alpha<\frac{1}{2}
\end{array}\right\}
$$

(ii) $\operatorname{MSE}\left(\hat{\bar{Y}}_{b k}\right)<\operatorname{MSE}\left(\hat{\bar{Y}}_{R}^{S T}\right)$ if

$$
\begin{aligned}
& \frac{\left(R_{U S 2}^{2} A_{1}+R_{U S 2} B_{1}\right)-\sqrt{\left(R_{U S 2}^{2} A_{1}+R_{U S 2} B_{1}\right)^{2}-R_{U S 2} A_{1} C_{1}^{*}}}{2 R_{U S 2}^{2} A_{1}}<\alpha< \\
& \frac{\left(R_{U S 2}^{2} A_{1}+R_{U S 2} B_{1}\right)+\sqrt{\left(R_{U S 2}^{2} A_{1}+R_{U S 2} B_{1}\right)^{2}-R_{U S 2} A_{1} C_{1}^{*}}}{2 R_{U S 2}^{2} A_{1}}
\end{aligned}
$$

(iii) $\operatorname{MSE}\left(\hat{\bar{Y}}_{b k}\right)<\operatorname{MSE}\left(\hat{\bar{Y}}_{S D}^{S T}\right)$ if

$$
\begin{aligned}
& \frac{\left(R_{U S 2}^{2} A_{1}+R_{U S 2} B_{1}\right)-\sqrt{\left(R_{U S 2}^{2} A_{1}+R_{U S 2} B_{1}\right)^{2}-R_{U S 2} A_{1} C_{2}^{*}}}{2 R_{U S 2}^{2} A_{1}}<\alpha< \\
& \frac{\left(R_{U S 2}^{2} A_{1}+R_{U S 2} B_{1}\right)+\sqrt{\left(R_{U S 2}^{2} A_{1}+R_{U S 2} B_{1}\right)^{2}-R_{U S 2} A_{1} C_{2}^{*}}}{2 R_{U S 2}^{2} A_{1}}
\end{aligned}
$$

(iv) $\operatorname{MSE}\left(\hat{\bar{Y}}_{b k}\right)<\operatorname{MSE}\left(\hat{\bar{Y}}_{S E}^{S T}\right)$ if

$$
\begin{aligned}
& \frac{\left(R_{U S 2}^{2} A_{1}+R_{U S 2} B_{1}\right)-\sqrt{\left(R_{U S 2}^{2} A_{1}+R_{U S 2} B_{1}\right)^{2}-R_{U S 2} A_{1} C_{3}^{*}}}{2 R_{U S 2}^{2} A_{1}}<\alpha< \\
& \frac{\left(R_{U S 2}^{2} A_{1}+R_{U S 2} B_{1}\right)+\sqrt{\left(R_{U S 2}^{2} A_{1}+R_{U S 2} B_{1}\right)^{2}-R_{U S 2} A_{1} C_{3}^{*}}}{2 R_{U S 2}^{2} A_{1}}
\end{aligned}
$$


(v) $\operatorname{MSE}\left(\hat{\bar{Y}}_{b k}\right)<\operatorname{MSE}\left(\hat{\bar{Y}}_{U S 1}^{S T}\right)$ if

$$
\begin{aligned}
& \frac{\left(R_{U S 2}^{2} A_{1}+R_{U S 2} B_{1}\right)-\sqrt{\left(R_{U S 2}^{2} A_{1}+R_{U S 2} B_{1}\right)^{2}-R_{U S 2} A_{1} C_{4}^{*}}}{2 R_{U S 2}^{2} A_{1}}<\alpha< \\
& \frac{\left(R_{U S 2}^{2} A_{1}+R_{U S 2} B_{1}\right)+\sqrt{\left(R_{U S 2}^{2} A_{1}+R_{U S 2} B_{1}\right)^{2}-R_{U S 2} A_{1} C_{4}^{*}}}{2 R_{U S 2}^{2} A_{1}}
\end{aligned}
$$

(vi) $\operatorname{MSE}\left(\hat{\bar{Y}}_{b k}\right)<\operatorname{MSE}\left(\hat{\bar{Y}}_{U S 2}^{S T}\right)$ if

$$
\begin{aligned}
& \frac{\left(R_{U S 2} A_{1}+B_{1}\right)-\sqrt{\left(R_{U S 2} A_{1}+B_{1}\right)^{2}-R_{U S 2} A_{1} C_{5}^{*}}}{2 R_{U S 2} A_{1}}<\alpha< \\
& \frac{\left(R_{U S 2} A_{1}+B_{1}\right)+\sqrt{\left(R_{U S 2} A_{1}+B_{1}\right)^{2}-R_{U S 2} A_{1} C_{5}^{*}}}{2 R_{U S 2} A_{1}}
\end{aligned}
$$

where

$$
\begin{aligned}
& A_{1}=\sum_{h=1}^{k} W_{h}^{2} \gamma_{h} C_{x h}^{2} S_{x h}^{2}, \quad B_{1}=\sum_{h=1}^{k} W_{h}^{2} \gamma_{h} C_{x h} S_{y x h}, \\
& A_{2}=\sum_{h=1}^{k} W_{h}^{2} \gamma_{h} S_{x h}^{2}, \quad B_{2}=\sum_{h=1}^{k} W_{h}^{2} \gamma_{h} S_{y x h}, \\
& C_{1}^{*}=R_{U S 2}^{2} A_{1}+2 R_{U S 2} B_{1}-R^{2} A_{2}+2 R B_{2}, \\
& C_{2}^{*}=R_{U S 2}^{2} A_{1}+2 R_{U S 2} B_{1}-R_{S D}^{2} A_{2}+2 R_{S D} B_{2}, \\
& C_{3}^{*}=R_{U S 2}^{2} A_{1}+2 R_{U S 2} B_{1}-R_{S E}^{2} A_{2}+2 R_{S E} B_{2}, \\
& C_{4}^{*}=R_{U S 2}^{2} A_{1}+2 R_{U S 2} B_{1}-R_{U S 1}^{2} A_{2}+2 R_{U S 1} B_{2}, \quad \text { and } \\
& C_{5}^{*}=R_{U S 2}^{2} A_{1}+2 B_{1}-R_{U S 2} A_{2}+2 B_{2} .
\end{aligned}
$$

(3.2), (3.3), (3.4), (3.5), (3.6) and (3.7) provides the regions of preference in which the suggested estimator is more efficient than the usual unbiased estimator, conventional ratio estimator, estimators $\hat{\bar{Y}}_{S D}^{S T}, \hat{\bar{Y}}_{S E}^{S T}, \hat{\bar{Y}}_{U S 1}^{S T}$ and $\hat{\bar{Y}}_{U S 2}^{S T}$ given by Kadilar and Cingi (2003). The range of $\alpha$ provides enough scope of choosing many estimators that are more efficient than the above considered estimators.

\section{Empirical Study}

To show the performance of the suggested estimator in comparison to other estimators, a natural population data set is being considered. The description of the population is given below.

\section{Population [Source: Singh and Mangat (1996), p.220]}

$Y$ : Total number of milch cows in 1993

$X$ : Total number of milch cows in 1990 


\begin{tabular}{lll}
\hline \hline & $N=4810 \& n=24$ & \\
\hline$n_{1}=7$ & $n_{2}=12$ & $n_{3}=5$ \\
$N_{1}=1260$ & $N_{2}=2400$ & $N_{3}=1150$ \\
$\bar{X}_{1}=15.29$ & $\bar{X}_{2}=17.25$ & $\bar{X}_{3}=17.8$ \\
$\bar{Y}_{1}=17.43$ & $\bar{Y}_{2}=20.42$ & $\bar{Y}_{3}=20.6$ \\
$\beta_{21}(x)=1.85$ & $\beta_{22}(x)=2.312$ & $\beta_{23}(x)=1.59$ \\
$C_{x_{1}}=0.2991$ & $C_{x_{2}}=0.3186$ & $C_{x_{3}}=0.1838$ \\
$S_{x_{1}}^{2}=20.905$ & $S_{x_{2}}^{2}=30.205$ & $S_{x_{3}}^{2}=10.7$ \\
$S_{y_{1}}^{2}=17.619$ & $S_{y_{2}}^{2}=18.386$ & $S_{y_{3}}^{2}=13.3$ \\
$S_{y x_{1}}=14.690$ & $S_{y x_{2}}=5.25$ & $S_{y x_{3}}=5.9$ \\
\hline
\end{tabular}

Table 1: Biases (\%) of different estimators

\begin{tabular}{|c|c|c|c|c|c|c|}
\hline Estimators & $\hat{\bar{Y}}_{R}^{S T}$ & $\hat{\bar{Y}}_{S D}^{S T}$ & $\hat{Y}_{S E}^{S T}$ & $\hat{\bar{Y}}_{U S 1}^{S T}$ & $\hat{\bar{Y}}_{U S 2}^{S T}$ & $\hat{\bar{Y}}_{b k}^{(o p t)} \alpha=0.71675$ \\
\hline Bias & 2.63 & 2.53 & 1.99 & 2.77 & 1.99 & 1.11 \\
\hline
\end{tabular}

Table 2: Percent relative efficiencies of $\hat{\bar{Y}}_{R}^{S T}, \hat{\bar{Y}}_{S D}^{S T}, \hat{Y}_{S E}^{S T}, \hat{\bar{Y}}_{U S 1}^{S T}, \hat{\bar{Y}}_{U S 2}^{S T}$ and $\hat{\bar{Y}}_{b k}^{(o p t)}$ with respect to $\bar{y}_{s t}$

\begin{tabular}{cccccccc}
\hline \hline Estimators & $\bar{y}_{s t}$ & $\hat{\hat{Y}}_{R}^{S T}$ & $\hat{\bar{Y}}_{S D}^{S T}$ & $\hat{Y}_{S E}^{S T}$ & $\hat{\bar{Y}}_{U S 1}^{S T}$ & $\hat{\bar{Y}}_{U S 2}^{S T}$ & $\hat{\bar{Y}}_{b k}^{(o p t)} \alpha=0.71675$ \\
\hline PREs & 100.00 & 62.96 & 64.55 & 74.12 & 119.54 & 11.37 & 121.81 \\
\hline \hline
\end{tabular}

Table 3: Range of $\alpha$ in which $\hat{\bar{Y}}_{b k}$ is more efficient $\bar{y}_{s t}, \hat{\bar{Y}}_{R}^{S T}, \hat{\bar{Y}}_{S D}^{S T}, \hat{Y}_{S E}^{S T}, \hat{\bar{Y}}_{U S 1}^{S T}$ and $\hat{\bar{Y}}_{U S 2}^{S T}$

\begin{tabular}{ccccccc}
\hline \hline Estimators & $\bar{y}_{s t}$ & $\hat{Y}_{R}^{S T}$ & $\hat{Y}_{S D}^{S T}$ & $\hat{Y}_{S E}^{S T}$ & $\hat{Y}_{U S 1}^{S T}$ & $\hat{Y}_{U S 2}^{S T}$ \\
\hline Range & $(0.50,0.9335)$ & $(-0.116,1.5498)$ & $(0.0835,1.350)$ & $(0.0981,1.335)$ & $(0.1373,1.296)$ & $(-0.730,2.163)$ \\
\hline
\end{tabular}

From Table 1, which reveals the bias in percent of different estimators, it is observed that the bias of the suggested estimator at optimum $\alpha$ is minimum.

Table 2 the shows that the largest gain in efficiency is due to the suggested optimum estimator $\hat{\bar{Y}}_{b k}$ over the unbiased estimator $\bar{y}_{s t}$, combined ratio estimator $\hat{\bar{Y}}_{R}^{S T}$, and estimators $\hat{\bar{Y}}_{S D}^{S T}, \hat{Y}_{S E}^{S T}, \hat{\bar{Y}}_{U S 1}^{S T}$ and $\hat{\bar{Y}}_{U S 2}^{S T}$ given by Kadilar and Cingi (2003). This implies that the gain in efficiency due to the proposed class of estimators $\hat{\bar{Y}}_{b k}$ can be obtained even when the scalar $\alpha$ deviates from its exact optimum value $\alpha_{(o p t)}$.

Table 3 exhibits the range of $\alpha$ in which the suggested estimator is more efficient than other estimators. We further note from Table 3, that there is enough scope in selecting the value of scalar $\alpha$ to obtain better estimators from the suggested class of estimators $\hat{\bar{Y}}_{b k}$.

\section{Conclusion}

Our empirical study shows that the proposed class of estimators provides estimators that are less biased and more efficient than other considered estimators. It gives the freedom to choose more efficient estimators, even if $\alpha$ deviates from its optimum value. Thus the suggested class of estimators is recommended for its use in practice.

\section{Acknowledgements}

Authors are thankful to the referees for their valuable suggestions regarding the improvement of the paper. 


\section{References}

Cochran, W. G. (1940). The estimation of the yields of the cereal experiments by sampling for the ratio of grain to total produce, The Journal of Agricultural Science, 30, 262-275.

Kadilar, C. and Cingi, H. (2003). Ratio estimators in stratified random sampling, Biometrical Journal, 45, 218-225.

Murthy, M. N. (1964). Product method of estimation, The Indian Journal of Statistics, Series A, bf 26 69-74.

Pandey, B. N. and Dubey, V. (1988). Modified product estimator using coefficient of variation of auxiliary variate, Assam Statistical Review, 2, 64-66.

Robson, D. S. (1957). Application of multivariate polykays to the theory of unbiased ratio-type estimation, Journal of the American Statistical Association, 52, 511-522.

Singh, H. P. and Espejo, M. R. (2003). On linear regression and ratio-product estimation of a finite population mean, Journal of the Royal Statistical Society Series D. The Statistician, 52, 59-67.

Singh, R. and Mangat, N. S. (1996). Elements of Survey Sampling, Kluwer Academic Publishers, Boston.

Singh, H. P. and Tailor, R. (2005). Estimation of finite population mean using known correlation coefficient between auxiliary characters, Statistica, 65, 407-418.

Singh, H. P., Tailor, R., Tailor, R. and Kakran, M. (2004). An improved estimation of population mean using power transformation, Journal Indian Society Agricultural Statistics, 58, 223-230.

Sisodia, B. V. S. and Dwivedi, V. K. (1981). A modified ratio estimator using coefficient of variation of auxiliary variable, Journal Indian Society Agricultural Statistics, 33, 13-18.

Tailor, R. (2009). A modified ratio-cum-product estimator of finite population mean in stratified random sampling, Data Science Journal, 8, 182-189 (on line).

Tailor R. and Sharma, B. K. (2009). A modified ratio-cum-product estimator of finite population mean using known coefficient of variation and coefficient of kurtosis, Statistics in Transition, 10, $15-24$.

Upadhyaya, L. N. and Singh, H. P. (1999). Use of transformed auxiliary variable in estimating the finite population mean, Biometrical Journal, 41, 627-636. 